\title{
Spinal injury associations: a British perspective
}

\section{J Hart}

Chairman, Spinal Injuries Association, Newpoint House, 76 St James's Lane, London N10 3DF, England.

It is solely from this island's experience that I shall dare to comment, as there are other organisations around the world that are striving to achieve similar objectives to those of the London-based Spinal Injuries Association (SIA), of which I am chairman, assisting paralysed people in leading an every day life as our motto suggests: '. . . because life doesn't stop when you're paralysed ....'

The SIA is the largest UK consumer organisation representing spinal cord injured (SCI) people. What better than specific organisations, run exclusively by SCI people to offer advice and support?

SIA was founded in 1974 and has grown to have a staff of 30 and part-time researchers with 6000 members, including people from 40 different countries. It is a member of the international movement, Disabled People's International. We have links with other similar organisations, some of which we have been instrumental in establishing, for example in Cyprus and in Nigeria.

Perhaps it is better to start by saying what our Association does not do - and why. SIA is not a grant making body, giving lump sums to individuals. It believes in helping people to help themselves, but at the same time not stepping in to provide where really the state should be providing.

Many of the things that SIA has had done or has had influence over highlight the modern accepted view that disabled people need to speak with their own voices on matters that affect them. For instance SIA commented on design briefs for spinal units, was responsible for a helicopter pad being build at one of the spinal units, and commissioned a survey into the design of toilets for disabled people.

Organisations such as SIA are important because they can offer experience and guidance based on people's successes and failures and give support and advice to others. Our newsletter is a medium for this, with helpful and interesting articles, and members can speak to other members through the link schemes for SCI people and their relatives.

Independent living, to which every SCI person has a right, is paramount in the ethos of our organisation. This means we are somewhat of a pressure group, pushing, along with the rest of the disability movement, for rights for disabled people. For instance in 1979 SIA managed to get 'until 70' driving licenses for SCI people and at the moment is fighting to keep the much used and needed Independent Living Fund intact. SIA employs a parliamentary adviser to assist members of the British Parliament in both Houses and as a result SIA has had several mentions in Hansard. We have members in high places, too, such as Baroness Masham and others in the House of Lords.

Although we generally leave research to other organisations SIA is concerned with areas important to SCI people, such as shoulder pain and root pain. It has produced reports on ageing with SCI, the personal and social implications of SCI and its effect on other family members. SIA also researched and produced a suppository inserter which, unlike its American counterpart, accommodates all different kinds of suppositories.

We entered the publication field many years ago. For instance we published, So You're Paralysed, the internationally revered 'first' book, which is translated into 5 different languages; World Wheelchair Traveller; and the care books: 2 booklets to assist staff in non specialist units, district nurses, doctors in general practice and personal assistants in the initial and long term care of someone with a spinal cord 
injury.

Some issues regarding SCI are sensitive. At SIA no subject is taboo-the management of continence, sexuality and financial concerns are freely discussed through our welfare department, although an individual's confidentiality is always kept.

We employ an experienced counsellor who offers a professional counselling service to anyone feeling extreme emotions, and the counsellor is developing the service by training a number of our members in counselling.

The very specific needs of SCI people are met by SIA in some other important areas. For instance we provide a personal assistance service (24-hour cover, for a maximum of 2 weeks per year), essentially for a member whose personal assistant wants to take a break or is suddenly ill. Our personal assistants can also be provided to go on holiday or business trips with a member.

Information is vital, hence the existence of an information service answering questions about holidays, transport, etc. Complaints and other issues are taken up on members' behalf, and journalists are kept happy with statistics and lists of members to interview.

We believe all holiday resorts and hotels should be accessible to wheelchairs, but as yet not all of them are. SIA established the Hotel and Holiday Consortium which pro- duced minimum standards for hotels covered in Providing Accessible Accommodation. SIA has also designed and built the first 3 narrowboats that can be skippered from a wheelchair, which optimises the ethos of the organisation - independence.

International links enable organisations to learn from one another. We have had considerable contact with American and Australian organisations, especially in the area of awareness and prevention. Our development officer has liaised frequently with these countries' organisations and gleaned information about their prevention campaigns - paving the way for our own, initially a pilot schools project. Also, the headway that disabled groups in the States have made can be a source of encouragement and inspiration.

The personal injury claims service, for which SIA employs a paraplegic solicitor, can provide quantum reports for the courts on legal claims cases and can recommend suitable solicitors.

Lay organisations set up to assist SCI people can work with professionals to enable SCI people to take control of their lives, making them successful, contributing members of society.

If any individual or group of individuals would like any advice about setting up a similar organisation then they should not hesitate to contact SIA for help in doing so. 